\title{
Historias de dictadura. Primeros cuentos de Sergio Ramírez.
}

\section{Stories of Dictatorship. Sergio Ramirez's First Tales.}

\begin{abstract}
Resumen
Los primeros textos del escritor nicaragüense Sergio Ramírez presentan algunos elementos que conforman, a manera de mosaico, los principales rasgos de la vida individual y social durante la larga dictadura somocista (1936 - 1979). Poder, violencia, dependencia política, social y económica son algunas de las problemáticas que asoman y su tratamiento se acentúa o modifica entre una publicación y otra más allá del orden de aparición. Asimismo, en el marco de las luchas por la liberación encabezadas por el Frente Sandinista de Liberación Nacional (FSLN), se cuestiona la función del arte y del intelectual. Propongo una serie de cuentos incluidos en Cuentos (1963), Nuevos Cuentos (1969) y Charles Atlas también muere (1976) en la que reviso, en primera instancia, la postura en torno al arte y al intelectual que exponen mediante la autofiguración del autor. Luego, exploro algunos elementos que conforman esos mundos narrativos y que funcionan como denuncia político-social al mismo tiempo que imaginan y reivindican una nueva nación en sintonía con los postulados presentes en las luchas revolucionarias.
\end{abstract}

Palabras claves

Sergio Ramírez, cuentos, dictadura, violencia, poder.

\begin{abstract}
The first stories of the Nicaraguan writer Sergio Ramírez present elements that form the main features of individual and social life during the Somoza's dictatorship (1936 - 1979). Some of the problems that appear are power, violence, political, social and economic dependence. Their treatment is accentuated or modified between one publication and another. These texts also questioned the role of art and the intellectual within the liberation struggles led by the Sandinista National Liberation Front. I propose a series of stories included in Cuentos (1963), Nuevos Cuentos (1969) and Charles Atlas también muere (1976)
\end{abstract}


in wich I review the stance on art and the intellectual in this context by the autofiguration of the author. Then, I explore some elements that shape those narrative worlds. They function as a political-social denunciation, while they imagine and claim a new nation in tune with the postulates present in the revolutionary struggles.

Keywords Sergio Ramírez, tales, dictatorship, violance, power.

En mi país/desde hace un rato empezaron los militares/ a matar niños a golpear el cuerpo tierno/ de los niños a levantar en bayonetas/ por el aire a los recién nacidos. (Claribel Alegría - "Operación Herodes")

Sergio Ramírez es escritor y político, participa activamente en la lucha contra la dictadura somocista; exiliado en Costa Rica forma parte del Grupo de los 12 en 1977. Durante el gobierno revolucionario es uno de los miembros de la Junta de Reconstrucción Nacional y vicepresidente de Nicaragua electo en 1984. Al retorno de la democracia es Diputado de la Asamblea Nacional y, progresivamente, se aleja del Frente Sandinista de Liberación Nacional (FSLN) hasta la ruptura definitiva y renuncia a la política en 1996. En sus inicios como escritor publica poesías y cuentos pero luego se inclina, decididamente, hacia la narrativa ya que, según sus propias palabras:

Pronto descubrí que lo que yo quería era ser narrador porque necesitaba narrar. Más tarde me encontré en una entrevista algo que decía Isaac Bashevis Singer y me identifiqué con eso porque él decía que uno descubre que es un narrador cuando siente la necesidad imprescindible de narrar, de que el mundo no está bien si uno no cuenta lo que está 
viendo, lo que está sintiendo y que hay que contárselo a otros porque a lo mejor se lo están perdiendo [...] de esa necesidad nací como narrador. ${ }^{1}$

Sus primeros textos aparecen en la revista Ventana, publicación de la Universidad de León que da nombre también al movimiento literario juvenil entre 1960 y 1964. "El Frente Literario Ventana, y la revista, fueron entre otras muchas, la expresión novedosa de una generación de jóvenes que desde una perspectiva cultural y política asumió el reto de su tiempo, en la Nicaragua oscura sometida a la dictadura cruel e impúdica de la familia Somoza" (Ventana 4). En esa producción, principalmente de cuentos, son ineludibles las referencias, explícitas o metaforizadas, a la vida y la sociedad nicaragüenses en el contexto de la larga dictadura somocista. Asume el reto de su tiempo desde su rol de escritor, la pluma acompaña al fusil para denunciar los abusos del poder pero también para imaginar una nueva nación más justa y soberana.

Tiempo después, Ramírez, reflexiona acerca de la relación que se establece entre el político y el escritor en el contexto de la dictadura y la revolución nicaragüenses, entre quien imagina y quien organiza la patria revolucionaria. En el primer momento, "el intelectual, el instruido, el leído, el sabido, frente a un pueblo, pobre, oprimido, es el que sabe más" (Oficios 26). Recurre a la imagen del sajurín "esa palabra tan linda, heredada del árabe, el zahorí, el mago, el adivino, el que lo sabe todo, el de Las mil y una noches, el de las maravillas. Y en Nicaragua el escritor es como un zahorí" (26). Es una potente representación que otorga a la literatura y al letrado un lugar central como portavoz de las realidades de un pueblo bajo un régimen totalitario.

En ese contexto, su producción temprana está marcada por la actualidad, pero esboza una estrategia que atraviesa la escritura de sus novelas. El autor bucea

${ }^{1}$ Entrevista realizada al autor en su casa en Managua en febrero de 2012. Algunos fragmentos fueron publicados en el suplemento literario de La Gaceta de Tucumán el 25 de marzo de ese año. 
en la historia de su país y de América Latina para encontrar los pies musicales ${ }^{2}$ que le permiten crear los mundos ficcionales. Sus obras plantean una problemática relación entre historiografía y ficción. Posee diferentes modulaciones que van desde una somera referencia marco de la narración, hasta ocupar un lugar central donde el hecho histórico es fundamental para la construcción del mundo narrativo ${ }^{3}$. La literatura le permite recobrar grandes zonas del pasado que fueron desechadas por la leyenda nacional que legitima al Estado (Carmen Perilli). En la recuperación de elementos residuales ${ }^{4}$ de la historia y la cultura nicaragüenses, estos relatos imaginan una nueva nación ${ }^{5}$, posible sólo mediante la revolución.

Sus opciones estéticas se modifican aunque siempre encuadradas en una propuesta realista. Observo una primera posición más clásica que se puede anclar en la propuesta de la literatura comprometida ${ }^{6}$ en términos sartreanos ${ }^{7}$; luego

${ }^{2}$ En la entrevista que realicé al autor en Managua menciona que en las crónicas históricas halla cosas que son propias de la novela y que están allí como un pie musical. En las páginas olvidadas de la historia de Nicaragua y América Latina encuentra imágenes narrativas que funcionan como tal. Son puertas o ventanas atractivas que tiene la literatura para meterse.

${ }^{3} \mathrm{Si}$ bien estas modulaciones se advierten en muchas de sus obras, puedo señalar como ejemplo paradigmático De tropeles y tropelías (1972) donde aparece la figura de un dictador arquetípico que puede situarse en cualquier lugar de América Latina. La reedición de 1983 agrega una viñeta final que puede ser contextualizada durante el terremoto de Managua de 1972 y refiere los sucesos posteriores de reconstrucción, es decir ya no representa a un dictador arquetípico, sino concretamente a Anastasio "Tachito" Somoza. En las novelas, Sergio Ramírez profundiza esta estrategia hasta llegar, por ejemplo, a construir la historia novelada como si se tratara de un texto histórico con la inclusión de documentos y testimonios también ficticios en Sombras nada más (2003).

${ }^{4}$ Raymond Williams (1977) al tratar el tema de las formaciones culturales define lo residual como ciertas experiencias, significados y valores que no pueden ser expresados o verificados en términos de la cultura dominante pero son vividos y practicados sobre la base de un remanente de alguna formación o institución social y cultural anterior

5 Sigo la propuesta de Benedict Anderson quien postula en Comunidades Imaginadas (2007) entender la nación como una comunidad política imaginada inherentemente limitada y soberana. Imaginada porque sus miembros no conocen a todos sus compatriotas pero en ellos vive la imagen de su comunión. Limitada pues tiene fronteras finitas aunque elásticas; soberana, porque sueña con la libertad y la garantía y el emblema de esa libertad es el Estado soberano; como comunidad pues se concibe siempre como un compañerismo profundo y horizontal más allá de las desigualdades existentes.

${ }^{6}$ En el prólogo a la edición facsimilar de Ventana, el autor recupera algunos de los fundamentos de la publicación, sobre todo aquellos que plantean la relación entre literatura y política. Los que participaban eran escritores y dirigentes políticos que buscaban "la calidad imprescindible de toda obra verdaderamente renovadora realizada por jóvenes, pero buscando asideros reales y propios, 
atraviesa una etapa en la que apela a elementos de la cultura de masas; por último, propone una nueva forma de realismo que desdibuja, hasta casi borrar, el límite entre historia y ficción. La violencia atraviesa toda su obra y se presenta bajo la forma de alguna de las dos categorías planteadas por Walter Benjamin ${ }^{8}$, es decir como fundadora o conservadora de derecho. José Ángel Vargas Vargas advierte que en sus novelas la violencia está siempre asociada a la noción de poder tanto en un plano histórico (el del estado dictatorial y el del revolucionario) como en el humano donde

El primero permite el establecimiento de relaciones de ambivalencia con la ficción y remite a unas coordenadas cronotópicas bien definidas, pues se refieren a las múltiples formas como se ha manifestado el poder en Nicaragua y América Latina, al mismo tiempo que connota el fracaso de una épica que prometía un mundo utópico. En el segundo se observan los efectos del poder como una fuerza que altera todo comportamiento y expone la complejidad de aquel individuo, que poseyendo el poder y la gloria, se enfrenta a un destino fatal en el que no existen las explicaciones lógicas y se impone la amarga miseria humana de vivir para la muerte y de constituirse en un espejismo de sombras. (Vargas Vargas 53)

como punto de partida hacia lo universal" (Ventana 1990 6). Esta postura se reafirma en las sucesivas publicaciones de la revista.

${ }^{7}$ A mediados de siglo, Jean Paul Sartre (1950) defiende la función social de la literatura. Propugna la figura del escritor comprometido que contribuye a producir cambios en la sociedad. No puede evadirse de la realidad y es responsable tanto de lo que dice como de lo que calla, porque entre ambos está la libertad de elección. El autor reconoce que la literatura es un producto estético, por lo tanto, un escritor comprometido también piensa la forma en que se expresa.

${ }^{8}$ Walter Benjamin en "Para una crítica de la violencia" postula que se la misma fundamenta en alguna de estas dos categorías para ser considerada como tal. En este caso la conservadora estaría representada por la Guardia Nacional mientras que la fundadora de derecho por las luchas revolucionarias. 
Poder, violencia, dependencia político, social y económica son algunas de las problemáticas que asoman ya en sus primeros textos. Todas ellas conforman, a manera de mosaico, los principales rasgos de la vida individual y social en dictadura que estas obras sugieren y cuyo tratamiento se acentúa o modifica entre una publicación y otra más allá del orden de aparición. Propongo una serie de cuentos incluidos en Cuentos (1963), Nuevos Cuentos (1969) y Charles Atlas también muere (1976) en la que reviso, en primera instancia, la postura en torno al arte y a la función del intelectual en la sociedad que asume mediante la autofiguración del escritor. Luego, exploro algunos elementos que conforman esos mundos narrativos y que funcionan como denuncia político-social al mismo tiempo que imaginan y postulan una nueva nación en sintonía con los postulados presentes en las luchas revolucionarias del Frente Sandinista de Liberación Nacional (FSLN).

\section{El intelectual y el arte en la dictadura}

La función del arte y del intelectual es una inquietud de Ramírez concebida desde la fundación de la revista Ventana junto a Fernando Gordillo. Pese a que la postura asumida se esboza de manera colectiva en la publicación juvenil, es en uno de los cuentos de su primera colección, que llamativamente no apareció en la revista, donde se explicita y en el que se desarrollan algunos de los puntos en torno a la responsabilidad del escritor/político.

El cuento titulado "Félis concóloris" (1963) narra el retorno de Alejandro Humberto Tiosca R., "uno de los más eminentes lexicólogos del mundo, dedicado desde años atrás a su fructífera labor intelectual” (Ramírez Cuentos 25), a su pequeño país de dos millones de habitantes, país en el que "existía y existe injusticia social. Hay hambre y desnudez, grandes latifundios, monopolios, bajos 
salarios y en fin, todas esas cintas de colores con que se atan los discursos en las plazas públicas" (Cuentos 26).

Una vez establecidos personaje y contexto, el narrador, en primera persona, incorpora la pregunta guía de todo el relato. Se construye casi como un ensayo donde bosqueja sus teorizaciones, al mismo tiempo que proyecta su propia imagen en oposición a la del protagonista

¿qué le importa a un país miserable como éste y qué le importa a los diarios, casi siempre en busca de noticias sobre crímenes, robos, falsificaciones, huelgas, atentados políticos, revoluciones, extorsiones, violaciones, etc., etc., la llegada de un tipo que sabe mucha gramática y maneja las palabras como el mecánico su torno y el panadero su masa? Yo había pensado lo mismo y me movía y aún me mueve la inquietud por tanto desamparado que hay en este país. Y, precisamente, estaba redactando un discurso que sobre la mala distribución de la tierra iba a pronunciar ante un mitin de campesinos, cuando saqué con alguna violencia el papel donde lo escribía, para meter en mi máquina éste, en que pinto la historia de un señor que llega a su país. (Cuentos 26)

El personaje ocupa el centro de la escena mediática y la gente comienza a interesarse por su historia cuando obtiene un premio internacional que se otorga cada dos años a quienes sobresalen en el campo de las ciencias y las letras antes de su arribo al país. Esa aureola se irradia también en el pueblo "que aunque padece hambre tiene sus grandes ataques de histeria colectiva" (29).

A su llegada, el gramático se instala en un hotel de montaña y anuncia al corresponsal de una revista científica que va a crear la palabra más bella del idioma en homenaje a su país. El gobierno alquila el "hotelito", declara zona de silencio toda el área e instala "el laboratorio de la palabra" (Cuentos 33) custodiado por guardias armados para impedir el paso y que nadie pueda 
entorpecer su tarea. Pasado el tiempo, la palabra tan esperada no llega, y la atención sobre Tiosca disminuye, hasta que, al final en una pequeña referencia en una esquina de un diario, el narrador lee que el experimento fue suspendido para luego enterarse de que el lexicólogo, de tanto leer y buscar la belleza, perdió la razón y fue internado en un sanatorio mental, mientras en el pequeño país se instala el estado de sitio y el narrador retoma la escritura del discurso que debe pronunciar en un mitin de campesinos.

A lo largo del texto, mientras se narra la historia del gramático, las referencias a la situación social, política y económica del país se suceden y repiten. El narrador se interesa, inicialmente, por la historia de Tiosca a pesar de que se trata de un "hombre insignificante para la mayoría y más para mis compañeros de la Acción popular Radical, donde milito” (Ramírez Cuentos 28). Sin embargo, se cuestiona sobre la utilidad de esta curiosidad "pues la presente historia no influyó en nada sobre las condiciones sociales y económicas del país y creo que de volver a repetirse tampoco influiría" (29).

En un espacio cuyos rasgos distintivos pueden asimilarse a los de la Nicaragua somocista, se instalan narrador y personaje. La oposición entre ellos problematiza la figura del intelectual, su función en la sociedad y el lugar del arte, representado en la búsqueda de la palabra más bella del idioma.

Tiosca es un intelectual que dedica su vida al trabajo científico con el lenguaje, habla siete idiomas, posee una fabulosa biblioteca y es mundialmente reconocido a tal punto que su nombre aparece en el Diccionario Universal de la Lengua. El narrador se autofigura como un militante preocupado por la realidad del país, pero que, al mismo tiempo, es un hombre "de algunas inquietudes" convencido "de que los hombres inquietos tienen un hondo sentido de observación" (Cuentos 27). Además, se puede pensar en la figura del escritor ya que, en definitiva, es él quien va a relatar la historia del gramático. Todas estas características nos hablan de un intelectual, uno comprometido con una causa, que es consciente del valor de la palabra y la prosa al estilo sartreano. La disyuntiva es 
entre dos tipos de intelectuales (tradicional u orgánico ${ }^{9}$ ) y la función que cumple cada uno. El destino de los personajes es el que la resuelve: mientras uno enloquece, el otro es quien cuenta la historia con una opción estética encuadrada en el paradigma realista.

El conflicto central del relato es la búsqueda de la palabra más bella y es el motivo que problematiza la función del arte en el contexto descripto. El hecho es considerado de suma importancia tanto por el personaje que se dedica a la tarea, como para el narrador, quien ante la noticia de su posible aparición:

Tenía la ansiedad que sólo siento cuando las noticias son enormes. Así, cuando se rumora que ha estallado una revuelta y se ven pasar varios camiones llenos de soldados, siento una explosión interna que me obliga a permanecer con los ojos bien abiertos y comentando el asunto con la demás gente. Es lo mismo. Que se invente una nueva palabra, que estalle una revolución, que ocurra un accidente aéreo. (35)

Las disquisiciones en torno a cómo será esa palabra apuntan a la discusión del lugar de la belleza o la estética. Algunos se preguntan si será tan bella como Trilce de Vallejo, mientras otros se preocupan sobre su destino, si podrá remediar el hambre del pueblo o un nuevo modo de denominar la miseria y la injusticia. La técnica que utiliza el científico y que lo lleva a la locura, la de recortar letras de periódicos y pegarlas para que formen palabras y frases, remite indudablemente a la utilizada por los escritores dadaístas. Las referencias a una zona particular del

${ }^{9}$ Para Antonio Gramsci todos los hombres son intelectuales aunque no todos desempeñan esa función en la sociedad. Distingue dos tipos: los tradicionales y los orgánicos. Los primeros son aquellos que hacen las mismas cosas de generación en generación e incluyen sacerdotes, profesores y administradores. Los segundos se conectan con clases o empresas que se sirven de ellos, se implican en forma activa en la sociedad y luchan por cambiar mentes o mercados. Todo grupo social "establece junto a él, orgánicamente, uno o más tipos de intelectuales que le dan homogeneidad no sólo en el campo económico, sino también en el social y en el político" (Gramsci 21). Estos intelectuales deben tener la aptitud adecuada para organizar la sociedad desde sus instituciones y el organismo estatal. 
campo de la literatura que retoma la teoría del arte por el arte se opone a la quienes consideran su función social.

El conflicto se resuelve por medio de la pérdida de cordura del científico y la escritura de su historia por parte del narrador quien focaliza más en la realidad social del país a través de citas y datos estadísticos que en una preocupación por el lenguaje o la forma en que la realiza. Se inscribe en el paradigma opuesto al que el experimento plantea, es decir el de la literatura y el arte que cumplen una función particular, en este caso la denuncia político - social sobre las condiciones de existencia del pueblo nicaragüense. Las realidades enunciadas en "Félis concóloris" nuclean las que se despliegan en los demás cuentos de estas colecciones.

\section{Vivir en dictadura}

Poder y violencia son dos rasgos distintivos de la vida en el contexto de la dictadura y dos ejes constantes que se modulan de diferentes maneras en los primeros textos del escritor. "Nicaragua es Blanca", publicado en Nuevos Cuentos (1969), imagina la posibilidad de una Navidad blanca, hecho que revela la forma en que se tejen los hilos del poder. Un anciano meteorólogo realiza el pronóstico en el observatorio que por fuera parece más "un kiosco de refrescos con su baranda de hierro forjado terminando en lancetas y su cúpula de latón, y por dentro igual a una oficina de telegrafía con sus manipuladores y belinógrafos, y él en mangas de camisa y ligas en los antebrazos, su visera verde en la frente y el cuello cerrado por un botón de hueso, como el uniforme de los generales de la guerra de Mena" (Cuentos 87).

La imagen caricaturesca del personaje se contrapone al rigor científico con el que el anciano realiza los cálculos antes de informar al "Sr. Presidente" sobre el 
extraordinario hecho. Cuando "el hombre" ${ }^{10}$ recibe la noticia, inmediatamente encarcela al anciano a pan y agua por haberlo despertado y lo manda a liberar después de que el suceso se confirma, vía Washington, no sin antes informarle que, como se trata de una cuestión de política internacional, "oficialmente fueron los Estados Unidos los que descubrieron que va a caer nieve ¿OK?” (Cuentos 90 énfasis en la edición).

Las someras referencias temporales (los estudios de meteorología realizados durante el gobierno de Zelaya y la lectura que realiza de los números atrasados del Observador Internacional del Tiempo del segundo trimestre de 1929) ubican esta historia en la década del 30, probablemente después de la retirada de los marines. Observo, entonces, dos modulaciones relacionadas con la problemática del poder. La primera es la pregunta por el lugar donde reside el poder político real más allá de que la intervención militar ya ha finalizado. La segunda, radica sobre la forma en que el poder del Estado o de "el hombre" se sostiene.

La caracterización del lugar de trabajo establece un claro contraste con las herramientas tecnológicas que posee "el amigo del norte" en la mirada del poder político. Cederles el descubrimiento de la histórica nevada no sólo da cuenta de una dependencia en términos de decisiones de índole pública, sino de una admiración que se extiende a todos los niveles de la sociedad. En el discurso de inauguración del árbol de Navidad, el presidente sostiene que "está próximo el día, anuncian nuestros amigos del norte, en que recibiremos como bendición del cielo una nevada; así que ya no tendremos nada que envidiarle a los países avanzados del viejo continente y de Norteamérica" (91).

La nieve se identifica con el progreso y la pertenencia, siempre deseada, al pequeño círculo de los países avanzados, donde, los dos modelos a seguir, son los que históricamente intervinieron en la vida pública de Nicaragua. Con ella aparecen, además, los productos materiales que simbolizan ese progreso y

${ }^{10}$ Manera en que se nombra al dictador en diversas obras del autor escritas en este período. Entre ellas De tropeles y tropelías (1972) y ¿Te dio miedo la sangre? (1977). 
representan al sistema que sostienen: las chimeneas; la leña de abedul traída desde aserraderos en New Hampshire; los pavos congelados de Miami, las manzanas de California; esquíes y colchas eléctricas para las tiendas; los pulóveres y bufandas con los que se viste la gente a pesar del calor sofocante de Managua; y el inmenso abrigo de armiño obsequiado por la embajada de Canadá, manta escocesa y gorro de Mujik con los que se viste el Presidente para la ocasión.

La intervención y la dependencia política y económica, traducidas en términos culturales y sociales, son un eje central que atraviesa la tercera colección de esta serie conformada por dos cuentos "Charles Atlas también muere" (1976) y “A Jackie, con nuestro corazón” (1976).

El primero, situado durante la ocupación de los marines, relata la historia de un telegrafista de San Fernando en las Segovias encargado de transmitir los mensajes del Capitán Hatfield USMC quien llegó al mando de la patrulla de marinos con la misión de hacer bajar a Sandino de la montaña. El narrador protagonista entabla amistad con el Capitán, aprende inglés y recibe algunos favores a cambio de nombrar a las personas del pueblo que de una u otra manera están ligadas al guerrillero. Lo recuerda porque lo ayuda a conseguir el curso de tensión muscular por correspondencia de Charles Atlas que produce su transformación de un "alfeñique" al hombre más desarrollado del mundo; y luego, el viaje a Nueva York para conocer a su héroe en persona.

$\mathrm{Su}$ encuentro es una desilusión pues Atlas está gravemente enfermo y muere durante la entrevista que sostienen al intentar hacer una de las poses que su método propugnaba. Este texto critica la ocupación norteamericana y sus métodos, como el de la delación. Pero, al igual que el anterior, problematiza la relación de los nicaragüenses con un otro, en este caso extranjero, figurado en el ideal de imagen masculina, además la música, los cigarrillos y el idioma que aprende el personaje, entre otros. Todos estos elementos anhelados representan una cultura y un mundo ajenos del que desea formar parte y considera superiores a los propios, tal como las clases dominantes nicaragüenses miran lo extranjero. Idolatra a Charles Atlas quien personifica el ideal masculino y a su método por su 
eficacia para conseguirlo. La enfermedad y muerte los desmitifica. Dan cuenta de una mirada sesgada sobre "el otro" al mismo tiempo que prefiguran la posibilidad de derrota de una cultura y política consideradas, hasta entonces, invencibles.

“A Jackie, con nuestro corazón” se desarrolla a fines de los 60" o principios de la de 1970 después de la muerte de John F. Kennedy. Ante el anuncio de la visita de "Jackeline" Kennedy a Nicaragua, el cuento narra los preparativos para su recepción. Diversos clubes y organizaciones se disputan el honor de ser los organizadores, entre ellos Lions International Club y el Rotary International Club pero la responsabilidad recae sobre el Virginian Country Club, cuyo presidente narra la historia. Se trata de un exclusivo club social fundado por accionistas norteamericanos y nicaragüenses. Su primer presidente fue el Coronel Glenn J. Andrews quien había combatido "las hordas sandinistas" (162), pero luego se queda en el país al casarse con Amalita Balcáceres del Castillo de alta cuna granadina.

La llegada y los preparativos son los motivos disparadores para caracterizar la relación que establecen las tradicionales oligarquías conservadoras y liberales con la política y la cultura norteamericanas. Algunos de los miembros del club son abogados de las diversas compañías mineras o bananeras que existen en el país; graduados de universidades como Georgetown, viven en cottages y toman cocktalis. La ínfima referencia de la participación del primer presidente del club en la lucha contra Sandino, las actividades realizadas por sus miembros y el lenguaje que utilizan muestra, de forma irónica, el hecho de que la intervención no es sólo militar, ni fue a principios de siglo, sino que se extiende a todos los ámbitos y continúa en la actualidad.

Este cuento agrega otro rasgo de la conformación social durante la dictadura: la relación entre la oligarquía y el grupo social cercano al dictador. Durante los preparativos, el narrador se convierte en una de las personalidades más importantes del país hasta tal punto que participa de las fiestas del Presidente de la República. En esos eventos, le sugiere nombres de ministros y funcionarios para ser invitados a embarcarse ya que el Queen Elizabeth es demasiado grande 
para los miembros del club y sus familias. Por un módico precio acceden. La mirada y el tono irónico respecto a esto permiten leer la tesis que postula: más allá de las tensas relaciones entre las clases tradicionales y la asociada al poder del dictador, sin la anuencia de las primeras, la larga dictadura no se podría haber sostenido.

Charles Atlas muere, Jackie Kennedy nunca llega a las costas de Nicaragua, ni la nieve a la capital, como lo anunciaran desde el norte. Por el contrario "las bujías amarillas estaban ya encendidas en los rieles y la música de las procesiones de barrio y los cohetes se oían por todos los rumbos, como en una Navidad cualquiera" (92). Tres fracasos, tres imposibilidades que pueden pensarse, como el de una política y una sociedad que históricamente ha mirado y se ha guiado por modelos extranjeros. La contrapartida que subyace es la utopía propuesta por la revolución en marcha de una nación soberana y libre, frente al discurso de la dictadura del progreso mediante modelos foráneos. Así como también la necesidad de recuperar y valorar lo propio representado, por ejemplo, en el conocimiento acertado del viejo meteorólogo en el primer cuento.

La segunda modulación en torno al poder que mencioné con anterioridad aparece esbozada en "Nicaragua es blanca" (1969) y se desarrolla en profundidad en otros textos de la serie. Este primer cuento revela que la forma en que el Estado o "el hombre" se sostienen en el poder es mediante el miedo y la violencia señalados en el doble encarcelamiento del anciano por haber interrumpido el sueño del "hombre" con su llamada y porque la nieve nunca aparece a pesar de que sus cálculos eran acertados.

"Al rescate", cuento de la primera colección que apareció por primera vez en abril de 1962 en el número 12 de Ventana, expone una comunidad signada por el miedo frente a una amenaza. Se enuncia en términos de crímenes que luego se explicitan "las noticias de los crímenes eran horribles: unas veces les torturaban con descargas eléctricas hasta matarlas y otras, simplemente les hundían un cuchillo en el cuello hasta que toda la sangre corría pesadamente" (22) frente a los cuales "todos dejaban impasibles que estas cosas sucedieran, nadie hacía nada 
para evitarlo. Estaban amarrados por una tensa corriente que les frenaba y les volvía cobardes" (22). En este contexto, aparecen las dos hermanas quienes deciden evitar la muerte de su madre secuestrada la noche anterior, hecho al que, ni los más valientes del pueblo, se habrían atrevido y sólo motivadas por el amor y no el afán de gloria. Son conscientes de la dificultad de lo que se proponen puesto que los hombres pueden estar armados de cuchillos, revólveres y quizás también rifles.

Al llegar al lugar donde se van a producir la tortura y el asesinato deciden esperar a la vera del camino a la víctima y los victimarios. En el interín sienten un grito desgarrador, se acercan a la ventana y ven en los ojos de la madre cómo se le escapa la vida. El cuento crece en tensión hasta llegar al clímax que se produce en el asesinato. Asesta el golpe en el final con el elemento sorpresa que genera el efecto que propone Edgar A. Poe pues "Cuando subió la mañana el sol las encontró de regreso mordiendo el zacate seco de la vera del camino. Solas, tristes y huérfanas, las dos vacas hermanas llevaban nublados sus grandes corazones por la sangre derramada que tanto amaban" (24). El efecto detona la significación del cuento que potencia la metáfora de las hermanas que resultan ser dos vacas.

Este texto de la primera colección alegoriza la violencia y el miedo de la vida en dictadura al mismo tiempo que adelanta una de las representaciones recurrentes de la revolución sandinista en las obras del autor: la de una lucha desigual, al estilo de David y Goliat, motivada por el amor. A diferencia de todos los demás, el relato no posee ninguna referencia cronotópica que permita ubicarlo explícitamente. Sin embargo, tanto el contexto de producción como el marco en el que fue publicado permiten hacer esta lectura.

La problemática de la violencia va in crescendo en los textos de esta primera etapa de la producción de Sergio Ramírez. A la metáfora inicial le sigue una pequeña mención donde se escamotea parte de la información pero se establecen coordenadas temporales y espaciales específicas; hasta ocupar el centro de la escena en "El centerfield", cuento perteneciente a la segunda colección. Aquí se representa la violencia conservadora, es decir la estatal, ya que 
narra el interrogatorio y posterior asesinato de un ex beisbolista, preso por haber ayudado a su hijo "enmontañado" guardándole una valija con armas y, según la versión oficial, porque durante meses estuvo "pasando parque, armas cortas, fulminantes, panfletos” en su casa “dormían los enemigos del gobierno" (98).

El relato se construye en doble temporalidad y registro. Alterna escenas del pasado del protagonista mientras es interrogado por el capitán. Los dos registros se configuran a partir de la realidad palpable del cuarto del interrogatorio y el partido de beisbol que el personaje imagina cuando observa el espacio de la cárcel mientras esto sucede. El juego imaginario los aúna. Le permite modificar el resultado del partido de su vida con la selección que recuerda en todo momento y se transforma en el posible marco ideal para una fuga de la prisión.

El interrogatorio se compone de frases cortas que le dan un ritmo acelerado como si se tratara de una formalidad; la decisión de eliminarlo ya está tomada. Esta estrategia y las referencias al miedo que siente el personaje (del que se evade mediante el recuerdo) configuran el estado de violencia instalada y reconocida por todos, más que la referencia o descripción de hechos concretos que sí aparecen en las novelas del autor. Ante la pregunta del capitán sobre el tiempo que hace que no ve a su hijo, "Lo miró al rostro y sacó de nuevo su pañuelo. / - Usted sabe que ya lo mataron. ¿Por qué me pregunta?” (99). Esta respuesta reafirma esa hipótesis.

En el final, el sargento pregunta al capitán sobre lo que debe poner en el parte sin haber escuchado siquiera el interrogatorio. Prefigura un estado donde la justicia está ausente y donde el destino de las personas queda sujeto a la decisión arbitraria del dictador o sus subordinados. El capitán responde casi en términos exactos a la forma como el preso ha imaginado la posible huida "- Era beisbolista, así que invéntate cualquier babosada: que estaba jugando con los otros presos, que estaba de centerfield, que le llegó un batazo contra el muro, que aprovechó para subirse al almendro, que saltó la tapia, que corriendo en el solar del rastro lo tiramos" (99). Podría pensarse que, en el juego de espejos entre realidad e 
imaginación, se busca evidenciar los niveles de crueldad y violencia sostenidos por el aparato del Estado que igualan o superan lo imaginable.

\section{Algunas consideraciones finales}

Los primeros cuentos de Sergio Ramírez focalizan en las condiciones de vida en el marco de la dictadura somocista que se caracterizan por la violencia y el exceso de poder. En torno a la primera existe una clara distinción entre la institucional, la que se utiliza para la conservación del poder del régimen; y la revolucionaria, la que busca instituir o fundar una nueva sociedad de derecho. El segundo se manifiesta tanto en términos histórico - políticos como humanos. Alude a las diferentes maneras en las que el poder se manifiesta en América Latina, pero también cómo lo vive el ciudadano quien, ya sea por miedo o conveniencia, pierde su libertad y actúa servilmente en función de los deseo del dictador.

El escritor explora algunos rasgos constitutivos de la identidad nicaragüense en una serie de elementos que se replican y reproducen a lo largo de su historia. Identidad y alteridad son fundamentales en estos textos que evidencian que la histórica dependencia política y económica no termina con el fin de la ocupación militar, sino que se traduce en términos culturales y sociales. Con tono irónico, muestra cómo la sociedad vive con la mirada hacia el afuera y el otro. Las experiencias narradas son fallidas y representan el fracaso de una propuesta política que se sostiene desde la independencia. La utopía revolucionaria penetra por ese resquicio. Frente a la violencia, los excesos de poder y el fiasco de un modelo y un sistema social, la respuesta posible es la revolución.

Es clara la opción por una estética encuadrada en el paradigma realista y la introducción de técnicas y materiales provenientes de la cultura de masas así como modelos literarios propios de la tradición latinoamericana. Crea arquetipos 
que actúan como modelos de la revolución. La figura del escritor/político se torna central pues, al mismo tiempo que imagina una nueva nación, problematiza la forma en que se organiza, focalizando principalmente en el lugar que ocupa el arte.

Las colecciones de cuentos y relatos de la primera etapa de producción de Sergio Ramírez evidencian cambios no sólo en términos estéticos sino también en relación con las formas en que las diversas problemáticas (poder, violencia, función del arte) se representan. Es una escritura que pretende objetivar la realidad en función de una denuncia político-social al mismo tiempo que experimenta a partir del lenguaje sin abandonar la función social de la literatura.

\section{Bibliografía}

AAVV. Ventana: publicación de artes y letras de los estudiantes de la Universidad Nacional. Autónoma de Nicaragua. Managua: Editorial Nueva Nicaragua, 1990. http://www.sergioramirez.com/index.php/otros-temas-deinteres/305-coleccion-revista-ventana-1961-1963

Aguilar, Gonzalo. "Los intelectuales de la literatura: cambio social y narrativas de identidad". Historia de los intelectuales en América Latina. Tomo II. Carlos Altamirano, director.Bs. As.: Katz Editores, 2010. 685 - 711. Bajtin, Mijail. Estética de la creación verbal. Buenos Aires: Siglo XXI, 2011. Barbero, Jesús Martín. De los medios a las mediaciones, Comunicación, cultura y hegemonía. Universidad Autónoma Metropolitana, México: Anthropos, 2010. 
Benjamin, Walter. Estética y Política. Buenos Aires: Las Cuarenta, 2009.

Cherem, Silvia. Una vida por la palabra. Entrevista con Sergio Ramírez. México: Fondo de Cultura Económica, 2004.

Gilman, Claudia. Entre la pluma y el fusil. Buenos Aires: Siglo XXI Editores, 2003.

Gramsci, Antonio. La formación de los intelectuales. México: Editorial Grijalbo, 1967.

Perilli, Carmen. Historiografía y ficción en la Narrativa Hispanoamericana. Tucumán: Universidad Nacional de Tucumán, FFyL, 1995.

Poe, Edgar A.Método de composición. http://www.ciudadseva.com/textos/teoria/opin/metodo_de_composicion.htm

Rama, Ángel. La ciudad letrada. Montevideo: Fundación Internacional Ángel Rama, 1984.

Ramírez, Sergio. Cuentos Completos. México: Fondo de Cultura Económica, 2013.

---. Oficios compartidos. México: Siglo XXI Editores, 1994.

Ríos, María del Pilar. "No aspiro a ser un bestseller sino un longseller. Entrevista a Sergio Ramírez”, La Gaceta Literaria, 25 de marzo de 2012. Disponible en http://www.lagaceta.com.ar/nota/482693/LA-GACETA-Literaria/Noaspiro-a-ser-un-best-seller-sino-un-long-seller.html

Said, Edward. Representaciones del intelectual. Barcelona: Paidós, 1996.

Sartre, Jean-Paul. ¿Qué es la literatura?. Buenos Aires: Losada, 1950.

Urbina, Nicasio. "Violencia y poder en la obra de Sergio Ramírez". Otro Lunes. Revista Hispanoamericana de cultura (2009). http://otrolunes.com/archivos/09/html/unos-escriben/unos-escriben-n09a48-p01-2009.html 
CATEDRAL TomadA: Revista de crítica literaria latinoamericana / Journal of Latin American Literary Criticism Historias de dictadura. Primeros cuentos de Sergio Ramírez.

Vargas Vargas, José Ángel. "Sergio Ramírez: Poder y desencanto", Revista Pensamiento Actual 5/6 (2005): 49-54.

http://revistas.ucr.ac.cr/index.php/pensamiento-actual/article/view/6654

$(\infty))$ EY

ULIS D. Sale
New articles in this journal are licensed under a Creative Commons Attribution 4.0 United States License.

This site is published by the University Library System, University of Pittsburgh as part of its D-Scribe Digital Publishing Program and is cosponsored by the University of Pittsburgh Press. 